\title{
Experimental evolution
}

Heredity (2008) 100, 441-442; doi:10.1038/hdy.2008.19

Microcosms are used in ecology and evolution to shrink space and time to manageable scales. They have never been extensively used in ecology, because many ecologists doubt that any important features of large complex systems, such as lakes, can be profitably investigated in small chambers or vials (Carpenter, 1996). Consequently, ecology lacks any generally accepted model system to facilitate the cumulative growth of understanding of how communities work. Evolutionary biologists have been less skeptical, and at least three major schools have adopted microcosm methods. Perhaps the best known is the extensive exploration of the effects of artificial selection in Drosophila (and to a lesser extent in other organisms, such as mice). This has provided us with a much clearer understanding of how selection acts on quantitative variation (Barton and Keightley, 2002), but is inevitably limited to the evolutionary short term by the brief duration of practicable experiments. Microbiologists formed a second school from the 1960s to the 1980s that produced many beautiful studies of biochemical adaptation in bacteria (Mortlock, 1984), but this was never fully assimilated into mainstream evolutionary biology and finally succumbed to the advancing tide of genomics. The third school descended from early chemostat studies and retained an interest in distinctively evolutionary issues (Dykhuizen, 1990). It is this school that has expanded so greatly in the last 15 years, revitalizing the field of experimental evolution (Bell, 2008).

The characteristic feature of the field is the selection experiment, which in this context means allowing natural selection to operate under controlled conditions. A very simple experiment is to introduce an isogenic population of microbes into a vial supplied with liquid nutrient medium containing a single limiting resource, transferring an aliquot of the culture at regular intervals to a fresh vial. The population will usually become better adapted over time and the simplicity of the set-up allows the mechanism of evolution to be studied in detail. If a genetically well-known model organism has been used, it should be possible to identify precisely the sequence of mutations responsible for adaptation. In this issue, Ferenci describes how Escherichia coli populations evolve in glucose-limited chemostats. Even in such a simple system, many avenues of adaptation exist, but a common observation is the rapid spread of mutations at rpos, a transcriptional regulator that controls the expression of a substantial fraction of the genome and is involved in accommodation to stressful conditions. These are loss-offunction mutations that enhance nutrient transport at the cost of attenuating the stress response, a cost well worth paying in normal well-buffered medium at lab temperature.

Ideally, a repeatable evolutionary event, such as the spread of rpos mutations, should also be predictable from first principles: from a knowledge of gene products and their interactions, it should be possible to predict which genes will be altered, how they will be altered and in what order, in any defined environment. Our understanding of cell biology is as yet wholly inadequate for the task, but the smaller genomes of viruses might be more accessible. Bull and Molineux describe the most ambitious attempts to date in pursuit of this grail, using phage T7 of E. coli. Given the detailed knowledge of T7 genetics, it should be possible to introduce a specific genetic lesion and then predict what compensatory mutations will be fixed so as to restore the lost function. In many cases, these mutations were correctly identified and for very simple genomes, at least, a nearly complete understanding of adaptation seems to be within grasp.

At the same time, both Ferenci and Bull and Molineux report some puzzling anomalies. The spread of rpoS mutants in E. coli populations was not only highly sensitive to conditions of growth, as could readily be predicted, but also varied among strains in unexpected ways. In T7, some successful predictions were made, but other outcomes were unpredicted, and were often difficult to explain even after the event. There are other problems, too, for example, rpoS $S^{-}$mutants replace the ancestral $\mathrm{rpoS}^{+}$type almost, but not quite completely, and after a time $r p o S^{+}$genotypes may stage a come-back. The process of evolution, even of a single ancestral genotype in a vial, is more subtle and complex than appears at first sight.

The genetic basis of adaptation is emphatically not determined solely by the physiological effects of gene products, because these must be translated into evolutionary change by selection. There are, therefore, specifically evolutionary mechanisms that govern what kind of mutations are likely to be substituted, such as the tendency for mutations of small effect to contribute rather little to adaptation, despite their high frequency, because they are likely to be lost by demographic stochasticity or overtaken by mutations of larger effect. Colegrave and Collins pursue this line of thought by arguing that the capacity to evolve may itself be selected, despite reducing fitness in the short term. Mutator alleles are easily understood as loss-of-function mutations in DNA polymerase genes that spread to high frequency in asexual populations by hitch hiking with the occasional beneficial mutations that they generate. Beyond this, however, is the suggestion that some of the enzymes involved in DNA replication and repair have evolved so as to be able to adjust mutation rates appropriately in response to environmental stress. Many will find the suggestion that patterns of gene interaction themselves evolve to enhance their ability to evolve even more controversial.

In the simplest systems, alleles are imagined to be either deleterious or neutral or beneficial. These labels are dissolved by even a small tincture of ecological reality. MacLean extends the simple classical model by considering the outcome of competition between fermenting 
and respiring strains of yeast. Fermentation is a profligate and wasteful strategy that is favored by selection because the fermenting strain immediately grabs the lion's share of glucose, so that the more productive respiring strain is starved of substrate. Nevertheless, the fermenters do not take over the population, mainly because they produce ethanol as a by-product and are the first to suffer from its effects. How severely this represses the fermenters, however, depends on population density, because it is more difficult to shed ethanol in crowded cultures, where there is a high concentration of ethanol in the external medium. Moreover, the outcome of competition is quite different when spatially structured populations are set up by plating the cells on to solid medium. Fermenting cells are now surrounded by like neighbors in the same colony and thus intoxicate the local environment, whereas respirers can reap the fruits of their prudent utilization of resources in fermentation-free patches.

The picture is now becoming more complicated, not only because a single mutation may have manifold and opposed consequences but also because the outcome of selection is likely to be affected by the pattern of social interactions. These themes are taken up by Elena et al. in the context of plant viruses. Each species of host plant may present a different challenge, generating trade-offs that lead to selection for specialization. At the same time, the mode of transmission is likely to affect the evolution of virulence, with vertically transmitted viruses evolving to become more benign because their fitness is tightly coupled to that of their host lineage. More generally, we might expect any symbiont to limit the damage done to its host, provided that the symbiont population descends from the same common ancestor. This is an example of kin selection; conversely, when several genotypes simultaneously infect a host, rapid reproduction is likely to be selected even if this causes the collapse of the host. Buckling and Brockhurst put this rule under the microscope and point out that it may not hold when host exploitation depends on public goods, such as siderophores or extracellular enzymes. The main reason is that pathogen populations tend to be invaded by nonproducing cheats, which use public goods without contributing any.

At this point, the picture is becoming much more complicated, with many possible trade-offs and several layers of social interactions, modulated by the physical conditions of growth, even though the basic situation remains as simple as one or two ancestral genotypes inoculated into a vial or onto a plate. The real world is much more complicated still, of course. The average population of bacteria, yeast or algae lives in a hostile world, where there may be hundreds of substrates, none of them individually adequate for growth, thousands of enemies and rivals and continual change in conditions. The modern school of experimental evolution has done a good job in unlocking the black box of adaptation in simple laboratory microcosms. The papers in this issue of Heredity show the progress that has been made. The next major challenge is to explore evolutionary mechanics in more realistic microcosms. This will require a fusion of ecology and evolution to develop the next generation of ecologically realistic yet genetically accessible model systems.

\author{
G Bell \\ Biology Department, McGill University, \\ Montreal, Quebec, Canada \\ E-mail: graham.bell@mcgill.ca
}

\section{References}

Barton NH, Keightley PD (2002). Understanding quantitative genetic variation. Nat Rev Genet 3: 11-21.

Bell G (2008). Selection, 2nd edn. Oxford University Press: Oxford.

Carpenter SR (1996). Microcosm experiments have limited relevance for community and ecosystem ecology. Ecology 77: 677-680.

Dykhuizen DE (1990). Experimental studies of natural selection in bacteria. Ann Rev Ecol Syst 21: 373-398.

Mortlock RP (1984). Microorganisms as Model Systems for Studying Evolution. Plenum Press: New York. 